\title{
ADVANCED GEAR DESIGN OF SMS GROUP*
}

\author{
Marcus Vinicius Laudares Mendonça ${ }^{1}$
} Michael Legge ${ }^{2}$

\begin{abstract}
SMS calculates and improves tooth and flank profiles individually to realise a homogeneous pressure distribution over the complete load flank. With a tooth profile and flank profile according to AGMA, ISO or DIN standard the contact pressure varies along the load flank. To realize a homogeneous distribution of the contact pressure and to avoid a premature mesh, SMS improves the tooth profile and flank profile in three steps. First SMS is doing a preliminary calculation and design according ISO, DIN or AGMA standard, with safety factors based on more than 125 years of experience. Second SMS calculates and checks the local contact pressure at the load flank under consideration of shaft, gearing and housing deformation, as well as the bearing behaviour. Finally SMS calculates and designs an improved tooth profile and flank profile to receive a homogeneous contact pressure at the load flank. The result is an increase of the torque transmitting capacity of up to $30 \%$ of the SMS advanced gears with the same outer dimensions than traditional gears.
\end{abstract}

Keywords: Drive; Gear; Modernization; SMS.

1 Dipl.-Ing., Product Engineer, Service Division, SMS Siemag Equipamentos e Serviços Ltda., Vespasiano, MG, Brazil.

2 MSc, Manager Design Drives, Drive Components, Hot Rolling Mills Division, SMS Siemag AG, Hilchenbach, Dahlbruch, Alemanha. 


\section{INTRODUCTION}

Unlike other gear manufacturers, SMS calculates and improves tooth and flank profiles individually to realize a homogeneous pressure distribution over the complete load flank. With a tooth profile and flank profile according to AGMA, ISO or DIN standard the contact pressure varies along the load flank. This is because tooth bending and shaft torsion as well as displacements and deviations caused by the gear housing or the roller bearings creates a premature mesh and a inhomogeneous contact pressure under load condition. The area with the highest contact pressure is exactly the area where pitting's or cut-outs occur first at the teeth.

\section{METHOD AND RESULTS}

To realize a homogeneous distribution of the contact pressure and to avoid a premature mesh, SMS improves the tooth profile and flank profile in three steps.

Step 1:

Preliminary calculation and design according ISO, DIN or AGMA standard, with safety factors based on more than 125 years of experience in metallurgical plant and rolling mill technology.

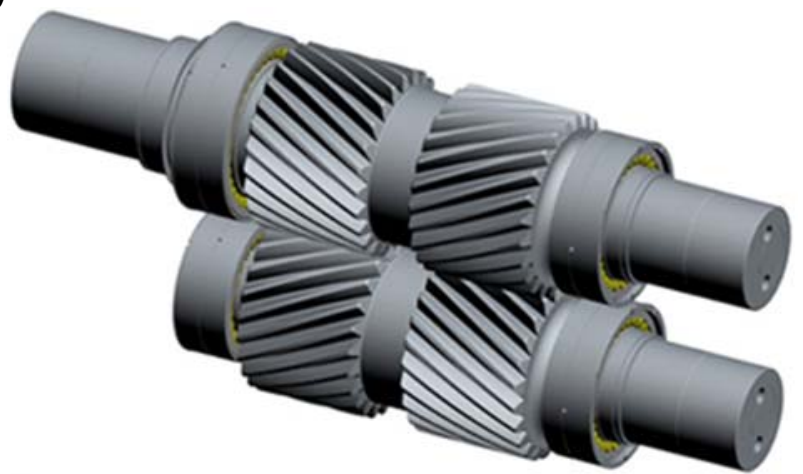

Figure 1. Mill pinions

Step 2:

Calculation and checking of the local contact pressure at the load flank. Influences such as shaft, gearing and housing deformation, as well as the bearing behavior (internal bearing geometry) are considered. Normally, the result is an inhomogeneous pressure distribution on the tooth profile and flank profile.

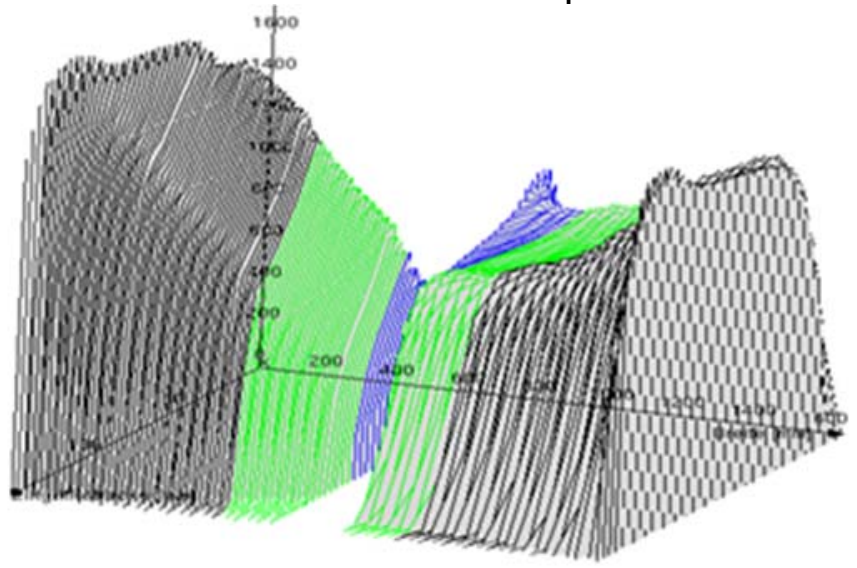

Figure 2. Local contact pressure at mill pinions with standard tooth profile and flank profile 
Step 3:

Calculation and design of an improved tooth profile and flank profile correction to compensate the deformations, like tooth bending and shaft torsion as well as displacements and deviations caused by the gear housing or the roller bearings, especially for the maximum drive train overload. The goal is a homogeneous contact pressure at the load flank to receive a high durability of the gear set.

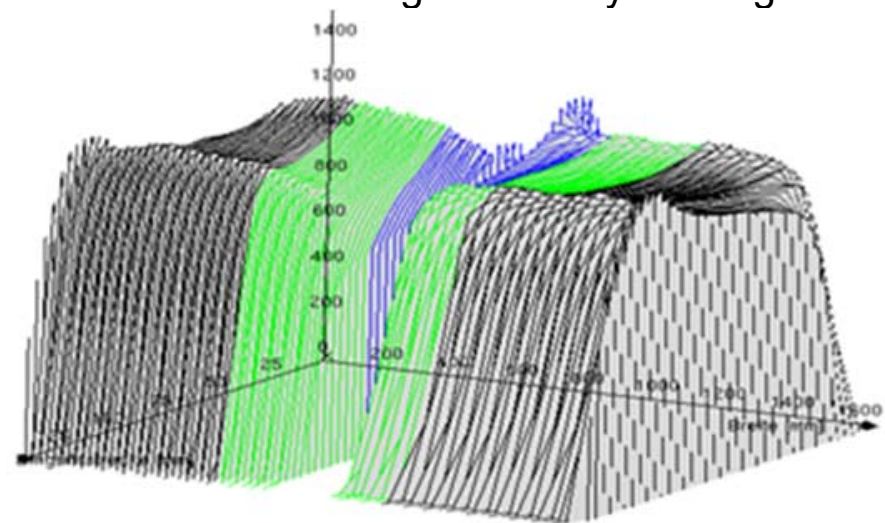

Figure 3. Local contact pressure at mill pinions with SMS advanced tooth profile and flank profile

\section{CONCLUSION}

What sets SMS advanced gear design apart from traditional gear design is that it increases the torque capacity of new gearboxes as well as existing gearboxes. Several customers have already replaced their existing gears with new gear sets based on the advanced gear design. That ensures that the new systems are capable of transmitting up to $30 \%$ higher torques with the same outer dimensions. 